\title{
Analysis of a Predator-Prey Model with Switching and Stage-Structure for Predator
}

\author{
T. Suebcharoen \\ Center of Excellence in Mathematics and Applied Mathematics, Department of Mathematics, Faculty of Science, Chiang Mai University, \\ Chiang Mai 50200, Thailand
}

Correspondence should be addressed to T. Suebcharoen; teeranush.s@gmail.com

Received 8 June 2017; Accepted 22 August 2017; Published 27 September 2017

Academic Editor: Yuji Liu

Copyright (C) 2017 T. Suebcharoen. This is an open access article distributed under the Creative Commons Attribution License, which permits unrestricted use, distribution, and reproduction in any medium, provided the original work is properly cited.

This paper studies the behavior of a predator-prey model with switching and stage-structure for predator. Bounded positive solution, equilibria, and stabilities are determined for the system of delay differential equation. By choosing the delay as a bifurcation parameter, it is shown that the positive equilibrium can be destabilized through a Hopf bifurcation. Some numerical simulations are also given to illustrate our results.

\section{Introduction}

The predator-prey system is important in dynamical population models and has been discussed by many authors [1-15].

In the related studies, a switching predator-prey model which has the switching property of predator was introduced by [7]. It was assumed that the predators catch prey in an abundant habitat. After a decrease in prey species population, the predator moves to another abundant habitat. In [8], the authors investigated a switching model of a two-prey onepredator system and they have shown that the system undergoes a Hopf bifurcation. They used the carrying capacity of prey as the bifurcations parameter. More examples on switching models can be found in [9-11]. Saito and Takeuchi [12] proposed a stage-structure model of a species' growth consisting of immature and mature individuals. It is assumed that the predators are divided into two-stage groups: juveniles and adults. Only the adult predators are able to catch prey species. As for the juvenile predators, they live with the adult predators. It is assumed that juveniles survive on prey already caught by adults. They live on a different resource which is available in the abundant habitat from the adult predators. Consequently, stage-structure model is more realistic than the model without stage-structure. In [14], it was further assumed that the time from juveniles to adults is itself state dependent. Qu and Wei [15] studied the asymptotic behavior of a predator-prey model with stage-structure. They found that an orbitally asymptotically stable periodic orbit exists in that model.

The purpose of the present paper is to study nonlinear delayed differential equations each of which describes a switching and stage structured predator-prey model. The present paper is organized as follows. In the next section, the main mathematical model is formulated and the positivity and boundedness of solutions are presented. In Section 3, we discuss the local stability of equilibria by analyzing the corresponding characteristic equations and we prove the existence of Hopf bifurcations for the model. Finally, numerical results and a brief discussion are provided.

\section{Model}

In this paper, we extend the switching predator-prey model in [8] by introducing stage structured with time delay into the model. We consider the switching with stage-structure predator-prey model of the following form:

$$
\begin{aligned}
& \frac{d x_{1}}{d t}=r x_{1}\left(1-\frac{x_{1}}{k}\right)+p q x_{2}-\frac{\beta x_{1} x_{2} y}{x_{1}+x_{2}} \\
& \frac{d x_{2}}{d t}=r x_{2}\left(1-\frac{x_{2}}{k}\right)+p q x_{1}-\frac{\beta x_{1} x_{2} y}{x_{1}+x_{2}}
\end{aligned}
$$




$$
\begin{aligned}
\frac{d y}{d t}= & 2 \delta \beta e^{-\gamma \tau} \frac{x_{1}(t-\tau) x_{2}(t-\tau) y(t-\tau)}{x_{1}(t-\tau)+x_{2}(t-\tau)}-\mu y \\
\frac{d y_{j}}{d t}= & 2 \delta \beta \frac{x_{1} x_{2} y}{x_{1}+x_{2}} \\
& -2 \delta \beta e^{-\gamma \tau} \frac{x_{1}(t-\tau) x_{2}(t-\tau) y(t-\tau)}{x_{1}(t-\tau)+x_{2}(t-\tau)} \\
& -\gamma y_{j}
\end{aligned}
$$

with initial conditions

$$
\begin{aligned}
& x_{1}(\theta), x_{2}(\theta), y(\theta), y_{j}(\theta) \geq 0 \quad \text { continuous on }[-\tau, 0), \\
& x_{1}(0), x_{2}(0), y(0)>0, 0 . \\
& y_{j}(0)>0 .
\end{aligned}
$$

The model is formulated under the following assumptions:

(1) It is assumed that two-prey species, denoted by $x_{1}$ and $x_{2}$, respectively, can be modelled by a logistic equation when the predator is absent. The parameter $r$ is the prey intrinsic growth rate and $k$ is its carrying capacity.

(2) The prey lives in two different habitats and each prey is able to migrate among two different habitats. The parameter $p$ is the probability of successful transition from each habitat and $q$ is inverse barrier strength in going out of the first habitat and the second habitat.

(3) The functions $\beta x_{1} /\left(x_{1}+x_{2}\right)$ and $\beta x_{2} /\left(x_{1}+x_{2}\right)$ have a characteristic property of a switching mechanism, where $\beta$ is capturing rate.

(4) The parameter $\delta$ is the rate of conversion of prey to predator and $\mu$ is the death rate of predator.

(5) The predators are derived into two-stage groups: juveniles and adults, which are divided by age $\tau$, and they are denoted by $y_{j}(t)$ and $y(t)$, respectively. It is assumed that juveniles take $\tau$ units of time to mature and $e^{-\gamma \tau}$ is the surviving rate of juveniles to adults. Notice, we assume that the juveniles suffer a mortality rate of $\gamma$.

For ecological reasons, we always assume that the initial data $x_{1}(\theta), x_{2}(\theta), y(\theta), y_{j}(\theta) \geq 0$ continuous on $[-\tau, 0)$, and $x_{1}(0)$, $x_{2}(0), y(0), y_{j}(0)>0$. If $\left(x_{1}(t), x_{2}(t), y(t), y_{j}(t)\right)$ is a solution of system (1) through that initial data, it is easy to verify that $\left(x_{1}(t), x_{2}(t), y(t), y_{j}(t)\right)$ is positive on the maximum existence interval of solution. Such solutions will be called positive solution. Moreover, if such a solution is bounded above and below, it is called a positive solution. Furthermore, we discuss the bounded positive solutions of system (1) which implies a natural restriction; that is, our system (1) must have a bounded positive solution. The following theorem guarantees that our stage-structure predator-prey model (1) with initial condition (2) always has a bounded solution. Therefore, every solution to system (1) is positive and bounded.

Theorem 1. Every solution of system (1) with initial condition (2) is bounded for all $t \geq 0$ and all of these solutions are ultimately bounded.

Proof. Let $V(t)=\gamma\left(\delta x_{1}+\delta x_{2}+y+y_{j}\right)$. By calculating the derivative of $V(t)$ with respect to $t$ along the positive solution of the system of system (1), we have

$$
\begin{aligned}
\dot{V}(t)= & \gamma \delta \dot{x}_{1}+\gamma \delta \dot{x}_{2}+\gamma \dot{y}+\gamma \dot{y}_{j} \\
= & \gamma \delta\left(r x_{1}-\frac{r}{k} x_{1}^{2}+p q x_{2}\right) \\
& +\gamma \delta\left(r x_{2}-\frac{r}{k} x_{2}^{2}+p q x_{1}\right)-\gamma \mu y-\gamma^{2} y_{j} .
\end{aligned}
$$

Let $\gamma>\mu$. We have

$$
\begin{aligned}
\dot{V}(t)+\mu V(t)= & (\gamma \delta r+p q+\gamma \mu \delta)\left(x_{1}+x_{2}\right) \\
& -\frac{\gamma \delta r}{k}\left(x_{1}^{2}+x_{2}^{2}\right)-\gamma(\gamma-\mu) y_{j} \\
< & (\gamma \delta r+p q+\gamma \mu \delta)\left(x_{1}+x_{2}\right) \\
& -\frac{\gamma \delta r}{k}\left(x_{1}^{2}+x_{2}^{2}\right) .
\end{aligned}
$$

Hence, there exists a positive constant $C$, such that

$$
\dot{V}(t)+\mu V(t) \leq C .
$$

Thus, we get

$$
V \leq\left(V(0)-\frac{C}{\mu}\right) e^{-\mu t}+\frac{C}{\mu} .
$$

Therefore, $V(t)$ is ultimately bounded; that is, each solution of system (1) is ultimately bounded.

\section{Local Stability and Existence of Hopf Bifurcation}

The main goal in this section is to investigate the stability of a positive equilibrium and the existence of a Hopf bifurcation.

Because of the last equation of system $(1), y_{j}(t)$ is completely determined by $x_{1}(t), x_{2}(t), y(t)$. Therefore, in the rest of this paper, we will study the following system:

$$
\begin{aligned}
& \frac{d x_{1}}{d t}=r x_{1}\left(1-\frac{x_{1}}{k}\right)+p q x_{2}-\frac{\beta x_{1} x_{2} y}{x_{1}+x_{2}} \\
& \frac{d x_{2}}{d t}=r x_{2}\left(1-\frac{x_{2}}{k}\right)+p q x_{1}-\frac{\beta x_{1} x_{2} y}{x_{1}+x_{2}}
\end{aligned}
$$




$$
\frac{d y}{d t}=2 \delta \beta e^{-\gamma \tau} \frac{x_{1}(t-\tau) x_{2}(t-\tau) y(t-\tau)}{x_{1}(t-\tau)+x_{2}(t-\tau)}-\mu y
$$

with the initial conditions $x_{1}(\theta), x_{2}(\theta), y(\theta) \geq 0$ continuous on $[-\tau, 0)$ and $x_{1}(0), x_{2}(0), y(0)>0$.

Before we proceed further, let us scale (7) by putting

$$
\begin{aligned}
\bar{x}_{1} & =\frac{x_{1}}{k}, \\
\bar{x}_{2} & =\frac{x_{2}}{k}, \\
\bar{y} & =e^{\gamma \tau} y \\
\alpha & =\frac{2 \delta \beta}{p q} k e^{-\gamma \tau} \\
g & =\frac{r}{p q}, \\
b & =\frac{e^{-\gamma \tau} \beta}{p q}, \\
d & =\frac{\mu}{p q}, \\
\bar{t} & =p q t, \\
\bar{\tau} & =p q \tau,
\end{aligned}
$$

and dropping the bars for the sake of simplicity. We obtain the following system containing dimensionless quantities:

$$
\begin{aligned}
& \frac{d x_{1}}{d t}=g x_{1}\left(1-x_{1}\right)+x_{2}-\frac{b x_{1} x_{2} y}{x_{1}+x_{2}} \\
& \frac{d x_{2}}{d t}=g x_{2}\left(1-x_{2}\right)+x_{1}-\frac{b x_{1} x_{2} y}{x_{1}+x_{2}} \\
& \frac{d y}{d t}=\alpha \frac{x_{1}(t-\tau) x_{2}(t-\tau) y(t-\tau)}{x_{1}(t-\tau)+x_{2}(t-\tau)}-d y .
\end{aligned}
$$

Next, we find equilibria of system (9) by equating the derivatives on the left-hand sides to zero. The equilibria are solutions of the system

$$
\begin{array}{r}
g x_{1}\left(1-x_{1}\right)+x_{2}-\frac{b x_{1} x_{2} y}{x_{1}+x_{2}}=0 \\
g x_{2}\left(1-x_{2}\right)+x_{1}-\frac{b x_{1} x_{2} y}{x_{1}+x_{2}}=0 \\
\alpha \frac{x_{1}(t-\tau) x_{2}(t-\tau) y(t-\tau)}{x_{1}(t-\tau)+x_{2}(t-\tau)}-d y=0 .
\end{array}
$$

This gives two possible equilibria which are

(i) boundary equilibrium $E_{1}=\left(x_{1}^{*}, g x_{1}^{*}\left(x_{1}^{*}-1\right), 0\right)$, which is corresponding to extinction of the predator, where $x_{1}^{*}>1$ is a real positive root of the cubic equation

$$
g^{3} x_{1}^{* 3}-2 g^{3} x_{1}^{* 2}+\left(g^{3}-g^{2}\right) x_{1}^{*}+\left(g^{2}-1\right)=0 \text {. }
$$

(ii) positive equilibrium $E_{2}=\left(\bar{x}_{1}, \bar{x}_{2}, \bar{y}\right)$, which is corresponding to coexistence of prey and predator and

$$
\begin{aligned}
\bar{x}_{1} & =\frac{d}{\alpha}(\bar{x}+1) \\
\bar{x}_{2} & =\frac{d}{\alpha \bar{x}}(\bar{x}+1) \\
\bar{y} & =\frac{\bar{x}+1}{b \bar{x}}\left(g\left(1-\bar{x}_{2}\right)+\bar{x}\right),
\end{aligned}
$$

Here $\bar{x}=\bar{x}_{1} / \bar{x}_{2}$ is a real positive root of the cubic equation

$$
g d \bar{x}^{3}+(g d-g \alpha+\alpha) \bar{x}^{2}+(g \alpha-\alpha-g d) \bar{x}-g d=0
$$

or

$$
(\bar{x}-1)\left(g d \bar{x}^{2}+(2 g d-g \alpha+\alpha) \bar{x}+g d\right)=0 .
$$

Obviously, $\bar{x}=1$ is the one real positive root of (13). The other two values of $\bar{x}$ will be real and positive if

$$
g>\frac{\alpha}{\alpha-4 d}
$$

We now analyze the stability of each equilibrium.

Let $E=\left(\widehat{x}_{1}, \widehat{x}_{2}, \hat{y}\right)$ be any arbitrary equilibrium. The characteristic equation about $E$ is given by

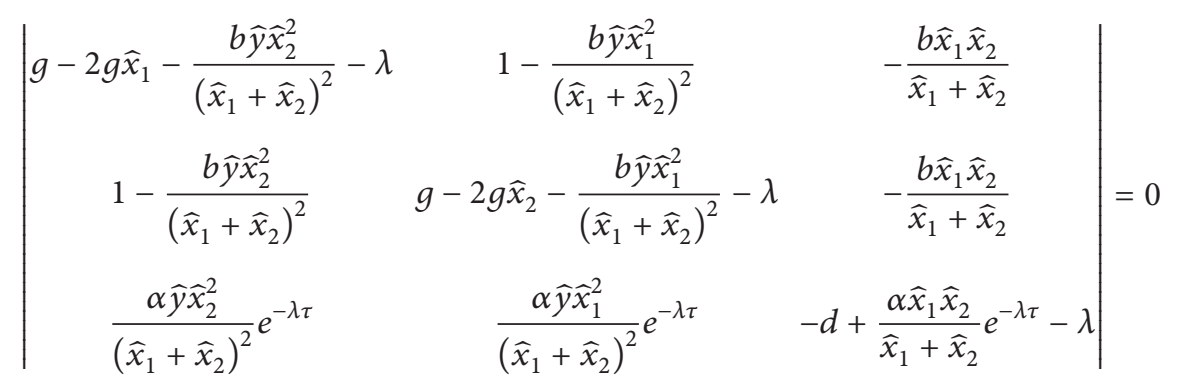


The next lemma gives conditions for the stability of equilibrium $E_{1}=\left(x_{1}^{*}, x_{2}^{*}, 0\right)$.

Theorem 2. The equilibrium $E_{1}=\left(x_{1}^{*}, x_{2}^{*}, 0\right)$ is

(i) unstable if $d<g \alpha x_{1}^{*}\left(x_{1}^{*}-1\right) /\left(1+g\left(x_{1}^{*}-1\right)\right)$;

(ii) locally asymptotically stable if $d>g \alpha x_{1}^{*}\left(x_{1}^{*}-1\right) /(1+$ $\left.g\left(x_{1}^{*}-1\right)\right)$.

Proof. We consider the characteristic equation of (16) at the equilibrium $E_{1}$. It follows that

$$
\begin{aligned}
& \left(\lambda+d-g \alpha \frac{x_{1}^{*}\left(x_{1}^{*}-1\right)}{1+g\left(x_{1}^{*}-1\right)} e^{-\lambda \tau}\right) \\
& \cdot\left(\left(g-2 g x_{1}^{*}-\lambda\right)\left(g-2 g^{2} x_{1}^{*}\left(x_{1}^{*}-1\right)-\lambda\right)-1\right) \\
& =0 .
\end{aligned}
$$

Hence, one characteristic root is the solution of the equation

$$
f_{1}(\lambda) \equiv \lambda+d-g \alpha \frac{x_{1}^{*}\left(x_{1}^{*}-1\right)}{1+g\left(x_{1}^{*}-1\right)} e^{-\lambda \tau}=0 .
$$

If $d<g \alpha x_{1}^{*}\left(x_{1}^{*}-1\right) /\left(1+g\left(x_{1}^{*}-1\right)\right)$, then $f_{1}(0)=$ $d-g \alpha\left(x_{1}^{*}\left(x_{1}^{*}-1\right) /\left(1+g\left(x_{1}^{*}-1\right)\right)\right)<0$, and $f_{1}(+\infty)=$ $\infty$. Therefore, $f_{1}(\lambda)$ has at least one positive root and the equilibrium $E_{1}$ is unstable. that is,

On the other hand, let $d>g \alpha x_{1}^{*}\left(x_{1}^{*}-1\right) /\left(1+g\left(x_{1}^{*}-1\right)\right)$;

$$
d-\frac{g \alpha x_{1}^{*}\left(x_{1}^{*}-1\right)}{1+g\left(x_{1}^{*}-1\right)}>0 .
$$

Then $f_{1}(-\infty)=-\infty$ and $f_{1}(0)>0$. Thus, a root of $f_{1}(\lambda)$ has negative real part. Hence, the other characteristic roots are the solution of the equation

$$
\left(g-2 g x_{1}^{*}-\lambda\right)\left(g-2 g^{2} x_{1}^{*}\left(x_{1}^{*}-1\right)-\lambda\right)-1=0
$$

that is,

$$
\begin{aligned}
f_{2}(\lambda) \equiv & \lambda^{2}+\left(x_{1}^{*}-1\right)\left(2 g+2 g^{2} x_{1}^{*}\right) \lambda+4 g^{3}\left(x_{1}^{*}\right)^{3} \\
& -6 g^{3}\left(x_{1}^{*}\right)^{2}+2\left(g^{3}-g^{2}\right) x_{1}^{*}+\left(g^{2}-1\right) \\
= & 0 .
\end{aligned}
$$

Since $x_{1}^{*}>1$ is a real positive root of the cubic equation $g^{3} x_{1}^{* 3}-2 g^{3} x_{1}^{* 2}+\left(g^{3}-g^{2}\right) x_{1}^{*}+\left(g^{2}-1\right)=0$, we have $\left(x_{1}^{*}-1\right)\left(2 g+2 g^{2} x_{1}^{*}\right)>0$. We, then, consider the last few terms from (21)

$$
\begin{aligned}
4 g^{3}( & \left.x_{1}^{*}\right)^{3}-6 g^{3}\left(x_{1}^{*}\right)^{2}+2\left(g^{3}-g^{2}\right) x_{1}^{*}+g^{2}-1 \\
= & \left(g^{3}\left(x_{1}^{*}\right)^{3}-2 g^{3}\left(x_{1}^{*}\right)^{2}+\left(g^{3}-g^{2}\right) x_{1}^{*}+g^{2}-1\right) \\
& +3 g^{3}\left(x_{1}^{*}\right)^{3}+\left(g^{3}-g^{2}\right) x_{1}^{*}-4 g^{3}\left(x_{1}^{*}\right)^{2} \\
= & \left(g^{3}\left(x_{1}^{*}\right)^{3}-2 g^{3}\left(x_{1}^{*}\right)^{2}+\left(g^{3}-g^{2}\right) x_{1}^{*}\right) \\
& +2 g^{3}\left(x_{1}^{*}\right)^{2}\left(x_{1}^{*}-1\right) \\
= & -\left(g^{2}-1\right)+2 g^{3}\left(x_{1}^{*}\right)^{2}\left(x_{1}^{*}-1\right) \\
= & g^{2}\left(2 x_{1}^{*} x_{2}^{*}-1\right)+1>0 .
\end{aligned}
$$

Thus, all the roots of characteristic equation have negative real part. The equilibrium $E_{1}$ is locally asymptotically stable.

Now, we analyze the stability of positive equilibrium $E_{2}\left(\bar{x}_{1}, \bar{x}_{2}, \bar{y}\right)$. The associated characteristic equation is

$$
\begin{aligned}
G(\lambda)= & \lambda^{3}+a_{1} \lambda^{2}+a_{2} \lambda+\left(a_{3} \lambda^{2}+a_{4} \lambda+a_{5}\right) e^{-\lambda \tau} \\
& +a_{6}=0
\end{aligned}
$$

where
In the following, we study the Hopf bifurcation for system (9), using the time delay $\tau$ as the bifurcation parameter. We assume that $\lambda=i \omega(\omega>0)$ is a root of the characteristic equation (23). Then we get 


$$
\begin{aligned}
- & \omega^{3} i-\omega^{2} a_{1}+a_{2} \omega i \\
& +\left(-\omega^{2} a_{3}+a_{4} \omega i+a_{5}\right)(\cos \omega \tau-i \sin \omega \tau)+a_{6} \\
= & 0 .
\end{aligned}
$$

By separating real part and imaginary part, we obtain

$$
\begin{array}{r}
\left(a_{5}-a_{3} \omega^{2}\right) \cos \omega \tau+a_{4} \omega \sin \omega \tau=a_{1} \omega^{2}-a_{6} \\
\left(a_{4} \omega\right) \cos \omega \tau+\left(a_{3} \omega^{2}-a_{5}\right) \sin \omega \tau=\omega^{3}-a_{2} \omega .
\end{array}
$$

By squaring both sides of the equations and using the property that $\sin ^{2} \omega \tau+\cos ^{2} \omega \tau=1$, we can simplify the above equation. As a result,

$$
\begin{aligned}
\omega^{6}+ & \left(a_{1}^{2}-2 a_{2}-a_{3}^{2}\right) \omega^{4} \\
& +\left(a_{2}^{2}-2 a_{1} a_{6}+2 a_{3} a_{5}-a_{4}^{2}\right) \omega^{2}+\left(a_{6}^{2}-a_{5}^{2}\right)=0 .
\end{aligned}
$$

Denote $v=\omega^{2}, e_{1}=a_{1}^{2}-2 a_{2}-a_{3}^{2}, e_{2}=a_{2}^{2}-2 a_{1} a_{6}+2 a_{3} a_{5}-a_{4}^{2}$, and $e_{3}=a_{6}^{6}-a_{5}^{2}$. Then (27) becomes

$$
h(v)=v^{3}+e_{1} v^{2}+e_{2} v+e_{3}
$$

By the Routh-Hurwitz criterion, we conclude that if

$$
\begin{aligned}
a_{1}+a_{3} & >0 \\
a_{5}+a_{6} & >0 \\
\left(a_{1}+a_{3}\right)\left(a_{2}+a_{4}\right) & >a_{5}+a_{6},
\end{aligned}
$$

(23) has no positive real roots. Therefore, we get the following results.

Theorem 3. Suppose conditions in (29) hold and $e_{1}, e_{2}>0$, $e_{3} \geq 0$. Then the equilibrium $E_{2}$ is locally asymptotically stable.

Proof. For $h(v)$ defined in (28), we have

$$
\frac{d h(v)}{d v}=3 v^{2}+2 e_{1} v+e_{2}
$$

and the zeros of (30) are

$$
v_{1,2}=\frac{-e_{1} \pm \sqrt{e_{1}^{2}-3 e_{2}}}{3}
$$

If $e_{1}, e_{2}>0$, then $\sqrt{e_{1}^{2}-3 e_{2}}<e_{1}$. Hence, $v_{1}$ and $v_{2}$ are negative. Thus, $d h(v) / d v=0$ has no positive root. Since $h(0)=e_{3} \geq 0$, it follows that $h(v)=0$ has no positive roots. Therefore, the equilibrium $E_{2}$ is locally asymptotically stable.

Theorem 4. Suppose that conditions in (29) hold and that

(i) either $e_{3}<0$,

(ii) or $e_{3} \geq 0, e_{2}<0$, and $2 \omega_{0}^{6}+\left(a_{1}^{2}-2 a_{2}-2 a_{3}^{2}\right) \omega_{0}^{4}+2 a_{5}^{2}-$ $a_{6}^{2} \neq 0$,

where $\omega_{0}$ satisfies $G\left(i \omega_{0}\right)=0$ with $G$ given in (23). Then the equilibrium $E_{2}$ is locally asymptotically stable if $\tau<\tau_{0}$ and is unstable if $\tau>\tau_{0}$, where

$$
\begin{aligned}
\tau_{0} & =\frac{1}{\omega_{0}} \\
& \cdot \cos ^{-1}\left(\frac{\left(a_{4}-a_{1} a_{3}\right) \omega_{0}^{4}+\left(a_{1} a_{5}+a_{3} a_{6}-a_{2} a_{4}\right) \omega_{0}^{2}-a_{5} a_{6}}{a_{4}^{2} \omega_{0}^{2}+\left(a_{5}-a_{3} \omega_{0}^{2}\right)^{2}}\right) .
\end{aligned}
$$

Furthermore, when $\tau=\tau_{0}$, a Hopf bifurcation occurs; that is, a family of periodic solutions are bifurcated from $E_{2}$ as $\tau$ passes through the critical value $\tau_{0}$.

Proof. If $e_{3}<0$, then it follows from (28) that $h(0)<0$ and $\lim _{v \rightarrow \infty} h(v)=\infty$. Thus, (27) has at least one positive root. If $e_{2}<0$, then $v_{1}=\left(-e_{1}+\sqrt{e_{1}^{2}-3 e_{2}}\right) / 3$ is one positive root of $d h(v) / d v=0$. Since $h(0)=e_{3} \geq 0$, it follows that $h(v)=0$ has at least one positive root. As a consequence, (27) has a positive root $\omega_{0}$. This implies that the characteristic equation (23) has a pair of purely imaginary roots.

Let $u(\tau)=\eta(\tau)+i \omega(\tau)$ be the eigenvalue of (23) such that $\eta\left(\tau_{0}\right)=0$ and $\omega\left(\tau_{0}\right)=\omega_{0}$. If there exists $\omega_{0}>0$, such that $G(i \omega)=0$. Then by the first equation of (26), we have

$$
\begin{aligned}
& \cos \left(\omega_{0} \tau_{j}\right) \\
& =\frac{\left(a_{4}-a_{1} a_{3}\right) \omega_{0}^{4}+\left(a_{1} a_{5}+a_{3} a_{6}-a_{2} a_{4}\right) \omega_{0}^{2}-a_{5} a_{6}}{a_{4}^{2} \omega_{0}^{2}+\left(a_{5}-a_{3} \omega_{0}^{2}\right)^{2}},
\end{aligned}
$$

and then

$$
\begin{aligned}
& \tau_{j} \\
& =\cos ^{-1} \frac{\left(a_{4}-a_{1} a_{3}\right) \omega_{0}^{4}+\left(a_{1} a_{5}+a_{3} a_{6}-a_{2} a_{4}\right) \omega_{0}^{2}-a_{5} a_{6}}{a_{4}^{2} \omega_{0}^{2}+\left(a_{5}-a_{3} \omega_{0}^{2}\right)^{2}} \\
& \quad+\frac{2 \pi j}{\omega_{0}}, \quad j=0,1,2, \ldots
\end{aligned}
$$

By taking the derivative of the characteristic equation (23) with respect to $\tau$, we have

$$
\frac{d \lambda(\tau)}{d \tau}=\frac{\left(a_{3} \lambda^{3}+a_{4} \lambda^{2}+a_{5} \lambda\right) e^{-\lambda \tau}}{\left(3 \lambda^{2}+2 a_{1} \lambda+a_{2}\right)-\left(a_{3} \lambda^{2}+a_{4} \lambda+a_{5}\right) \tau e^{-\lambda \tau}+\left(2 a_{3} \lambda+a_{4}\right) e^{-\lambda \tau}}
$$



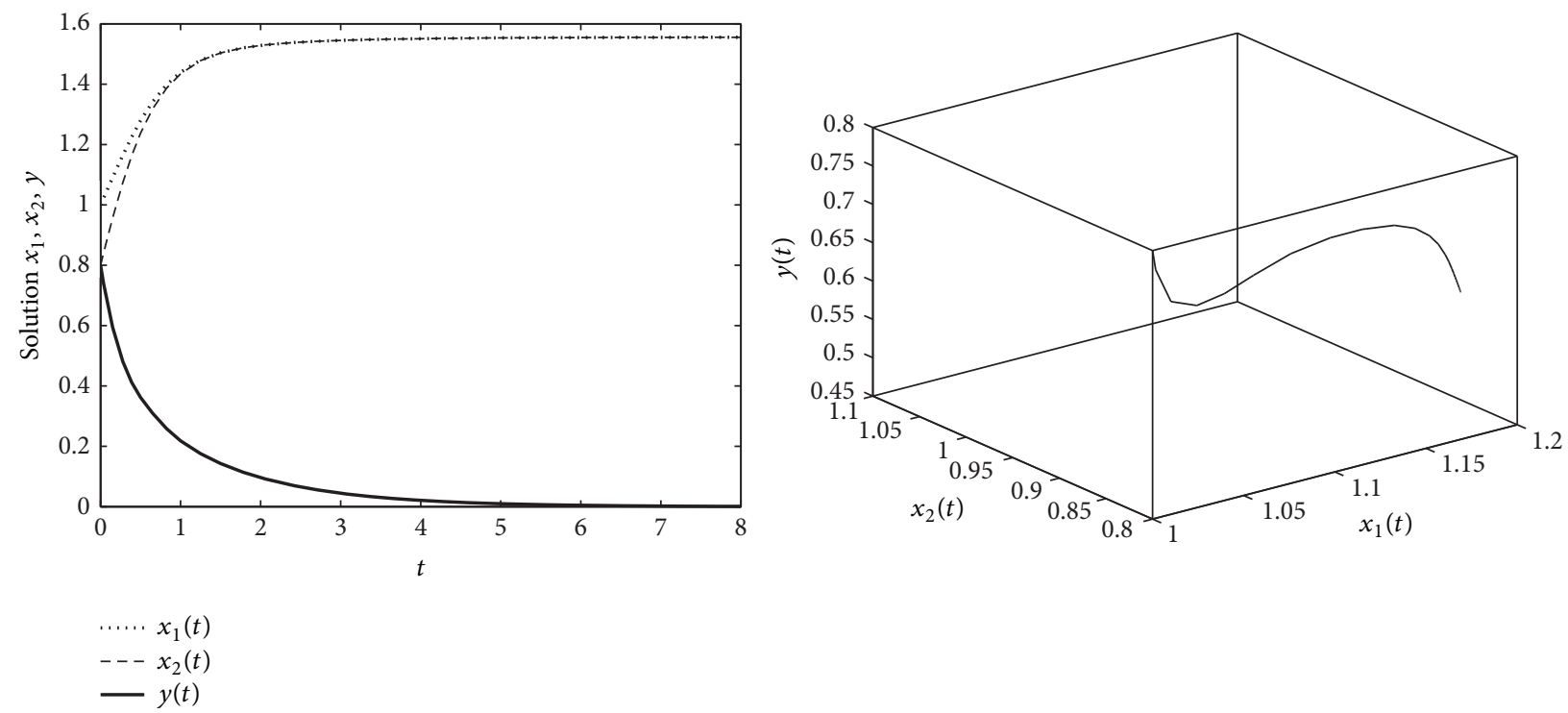

Figure 1: The behavior of $x_{1}, x_{2}$, and $y$ with respect to $t$ for Example 5 .

Thus,

$$
\begin{aligned}
\left(\frac{d \lambda(\tau)}{d \tau}\right)^{-1}= & \frac{\left(3 \lambda^{2}+2 a_{1} \lambda+a_{2}\right)+\left(2 a_{3} \lambda+a_{4}\right) e^{-\lambda \tau}}{\lambda\left(a_{3} \lambda^{2}+a_{4} \lambda+a_{5}\right) \tau e^{-\lambda \tau}} \\
& -\frac{\tau}{\lambda}
\end{aligned}
$$

We can also verify the following transversality condition [16]:

$$
\begin{aligned}
& \left(\left.\frac{d \operatorname{Re} \lambda(\tau)}{d \tau}\right|_{\tau=\tau_{0}}\right)^{-1} \\
& =\operatorname{Re}\left(\frac{\left(3 \lambda^{2}+2 a_{1} \lambda+a_{2}\right)+\left(2 a_{3} \lambda+a_{4}\right) e^{-\lambda \tau}}{\lambda\left(a_{3} \lambda^{2}+a_{4} \lambda+a_{5}\right) \tau e^{-\lambda \tau}}\right. \\
& \left.-\frac{\tau}{\lambda}\right)\left.\right|_{\tau=\tau_{0}} \\
& =\frac{2 \omega_{0}^{6}+\left(a_{1}^{2}-2 a_{2}-2 a_{3}^{2}\right) \omega_{0}^{4}+2 a_{5}^{2}-a_{6}^{2}}{\omega_{0}^{2}\left(\left(a_{5}-a_{3} \omega_{0}^{2}\right)^{2}+\left(a_{4} \omega_{0}\right)^{2}\right)} \neq 0 .
\end{aligned}
$$

Therefore, if $\tau=\tau_{0}$, then a Hopf bifurcation occurs; that is, a family of periodic solutions appear as $\tau$ passes through the critical value $\tau_{0}$.

\section{Numerical Simulations and Discussion}

In this section, we present some numerical simulation of system (9) at different parameters to illustrate our analytic results.
Example 5. Let $g=1.8 b=0.6 \alpha=2 d=3$ and we consider the following system:

$$
\begin{aligned}
\frac{d x_{1}}{d t} & =1.8 x_{1}\left(1-x_{1}\right)+x_{2}-\frac{0.6 x_{1} x_{2} y}{x_{1}+x_{2}} \\
\frac{d x_{2}}{d t} & =1.8 x_{2}\left(1-x_{2}\right)+x_{1}-\frac{0.6 x_{1} x_{2} y}{x_{1}+x_{2}} \\
\frac{d y}{d t} & =2 \frac{x_{1}(t-\tau) x_{2}(t-\tau) y(t-\tau)}{x_{1}(t-\tau)+x_{2}(t-\tau)}-3 y .
\end{aligned}
$$

In this case, we obtain only one boundary equilibrium $E_{1}=$ $(1.556,1.557,0)$, and the conditions of (ii) in Theorem 2 are satisfied. Therefore, the equilibrium $E_{1}$ is locally asymptotically stable. The behaviors of $x_{1}, x_{2}$, and $y$ with respect to $t$ are shown in Figure 1. According to the graph in Figure 1, the predator population decreases and eventually the predator species becomes extinct. As for prey species, the population of both species reaches the equilibrium as the predator population approaches zero.

Example 6. As an example, consider the following system:

$$
\begin{aligned}
\frac{d x_{1}}{d t} & =1.8 x_{1}\left(1-x_{1}\right)+x_{2}-\frac{0.6 x_{1} x_{2} y}{x_{1}+x_{2}} \\
\frac{d x_{2}}{d t} & =1.8 x_{2}\left(1-x_{2}\right)+x_{1}-\frac{0.6 x_{1} x_{2} y}{x_{1}+x_{2}} \\
\frac{d y}{d t} & =2 \frac{x_{1}(t-\tau) x_{2}(t-\tau) y(t-\tau)}{x_{1}(t-\tau)+x_{2}(t-\tau)}-0.3 y .
\end{aligned}
$$

There is a positive equilibrium $E_{2}=(0.3,0.3,7.53)$. By direct calculation, we have $e_{3}=-0.01903, \omega_{0}=0.639$, and $2 \omega_{0}^{6}+\left(a_{1}^{2}-2 a_{2}-2 a_{3}^{2}\right) \omega_{0}^{4}+2 a_{5}^{2}-a_{6}^{2}=0.2234 \neq 0$. From Theorem 4 , there is a critical value $\tau_{0}=1.1071$, and the 

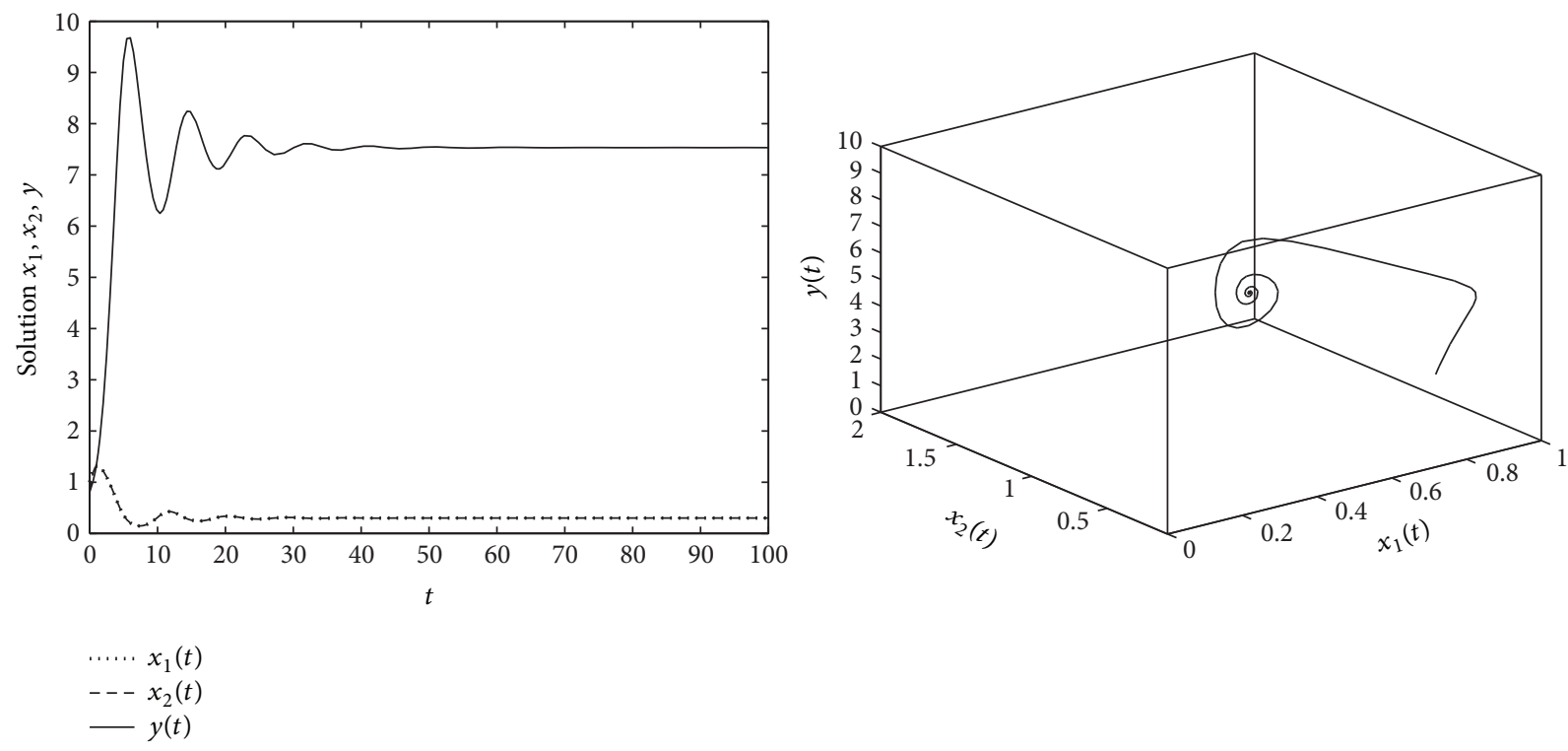

Figure 2: The behavior of $x_{1}, x_{2}$, and $y$ with respect to $t$ for Example 6 with $\tau=0.5$.
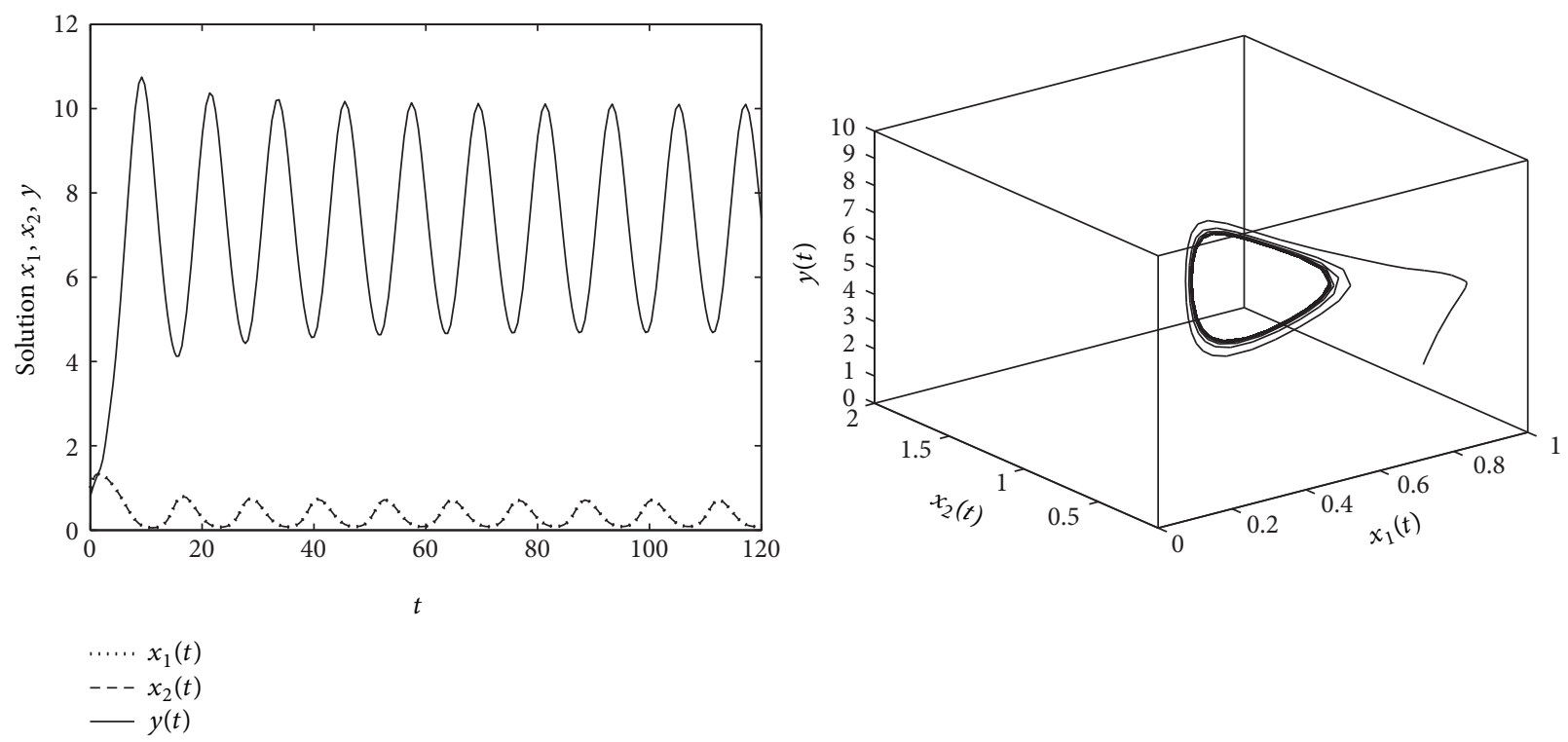

Figure 3: The behavior of $x_{1}, x_{2}$, and $y$ with respect to $t$ for Example 6 with $\tau=1.8$.

equilibrium $E_{2}$ is locally asymptotically stable as $\tau<\tau_{0}=$ 1.1071. A Hopf bifurcation occurs as $\tau=\tau_{0}=1.1071$ and the equilibrium becomes unstable and stable periodic solutions exist for $\tau>\tau_{0}=1.1071$. Figures 2 and 3 show the solutions of that system corresponding to $\tau=0.5$ and $\tau=1.8$. Furthermore, a bifurcation diagram for Example 6 is shown in Figure 4. This is an example when the predator and prey coexist permanently. If the time that juvenile takes to be mature is less than $\tau_{0}$, then both predators and prey population reach the nonzero equilibrium. They can coexist permanently. On the other hand, if the time that juvenile predators takes to become mature and ready to hunt is longer than $\tau_{0}$, then the population of both predator and prey species becomes unstable and periodic.

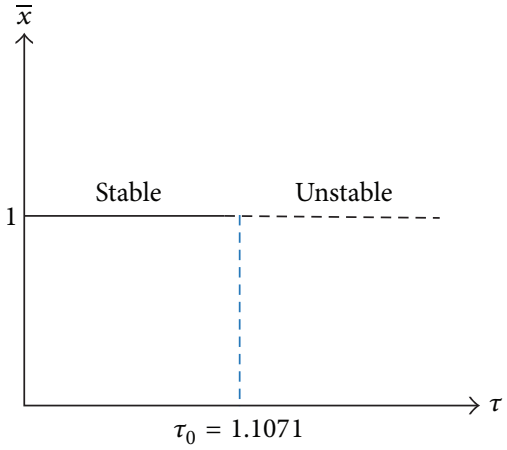

FIgURE 4: Bifurcation diagram for Example 6. 


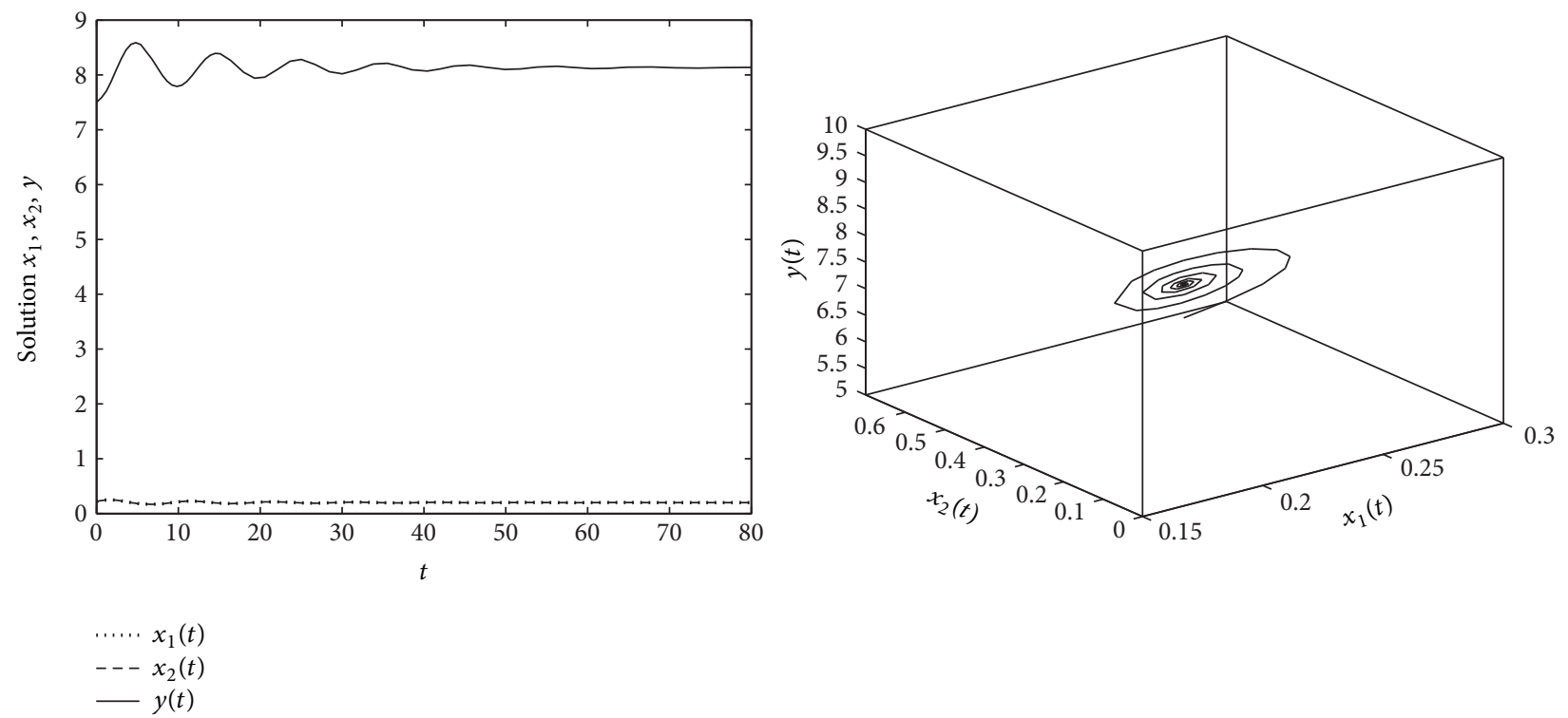

Figure 5: The behavior of $x_{1}, x_{2}$, and $y$ with respect to $t$ for equilibrium $E_{2}^{1}$ with $\tau=0.6$.
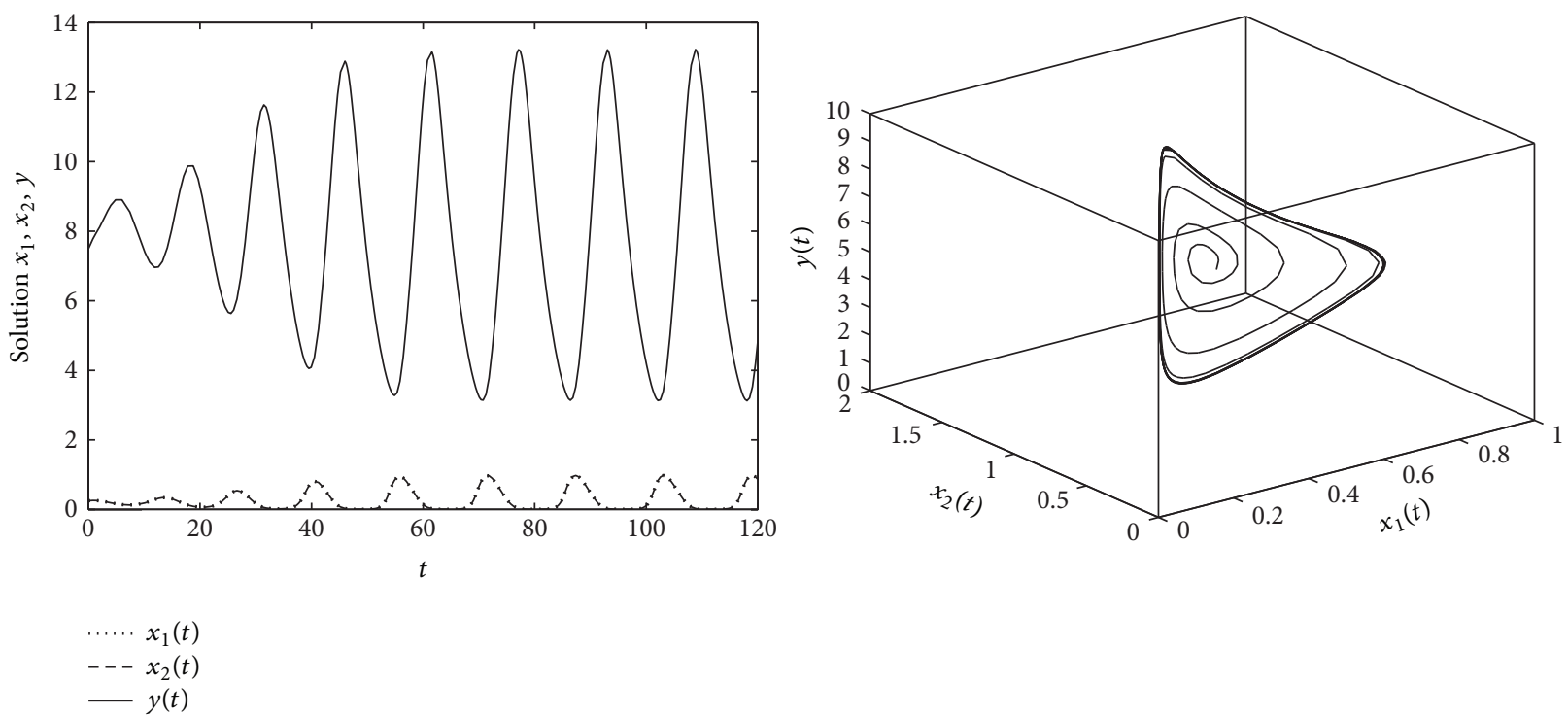

FIgURE 6: The behavior of $x_{1}, x_{2}$, and $y$ with respect to $t$ for equilibrium $E_{2}^{1}$ with $\tau=2$.

Example 7. As an example, consider the following system:

$$
\begin{aligned}
\frac{d x_{1}}{d t} & =1.8 x_{1}\left(1-x_{1}\right)+x_{2}-\frac{0.6 x_{1} x_{2} y}{x_{1}+x_{2}} \\
\frac{d x_{2}}{d t} & =1.8 x_{2}\left(1-x_{2}\right)+x_{1}-\frac{0.6 x_{1} x_{2} y}{x_{1}+x_{2}} \\
\frac{d y}{d t} & =2 \frac{x_{1}(t-\tau) x_{2}(t-\tau) y(t-\tau)}{x_{1}(t-\tau)+x_{2}(t-\tau)}-0.2 y .
\end{aligned}
$$

In this case, we obtain three positive equilibria $E_{2}^{1}=(0.2,0.2$, 8.133), $E_{2}^{2}=(0.1519,0.2927,8.7420)$, and $E_{2}^{3}=(0.2925$,
$0.1519,8.7410)$. By Theorem 4 , we know that the positive equilibrium $E_{2}^{1}$ is locally asymptotically stable when $\tau<\tau_{0}=$ 1.8227 and unstable when $\tau>\tau_{0}=1.8227$, and the system can also undergo a Hopf bifurcation at the equilibrium $E_{2}^{1}$ when $\tau$ crosses through the critical value $\tau>\tau_{0}=1.8227$; see Figures 5 and 6 . Similarly, at the positive equilibrium $E_{2}^{2}$, a Hopf bifurcation occurs as $\tau=\tau_{0}=2.4353$. Hence, the positive equilibrium $E_{2}^{2}$ is locally asymptotically stable when $\tau<\tau_{0}=2.4353$ and unstable when $\tau>\tau_{0}=2.4353$; see Figures 7 and 8 . For equilibrium $E_{2}^{3},(27)$ has no positive real root. Hence, equilibrium $E_{2}^{3}$ is locally asymptotically stable and no stability switches can occur; see Figure 9. On this last 

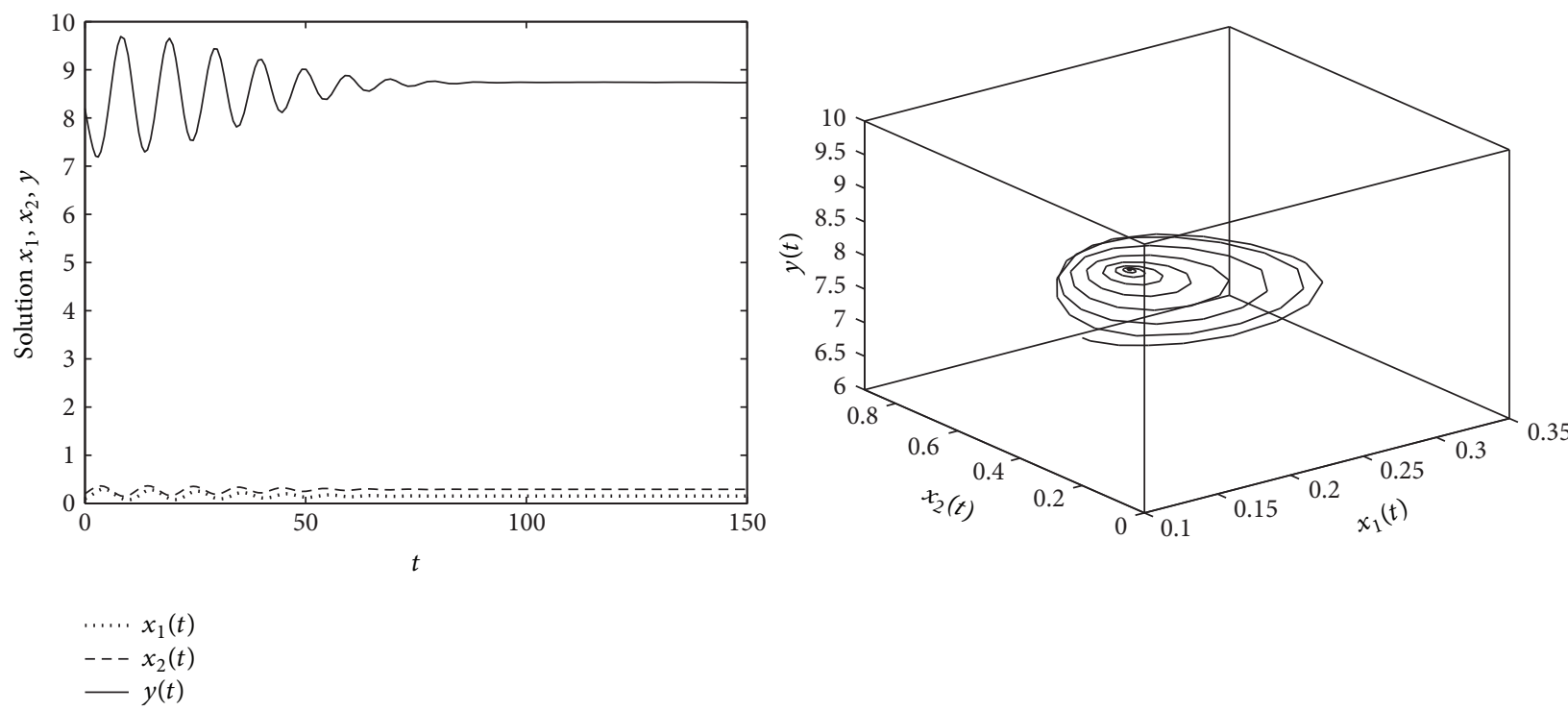

FIgURE 7: The behavior of $x_{1}, x_{2}$, and $y$ with respect to $t$ for equilibrium $E_{2}^{2}$ with $\tau=1.8$.
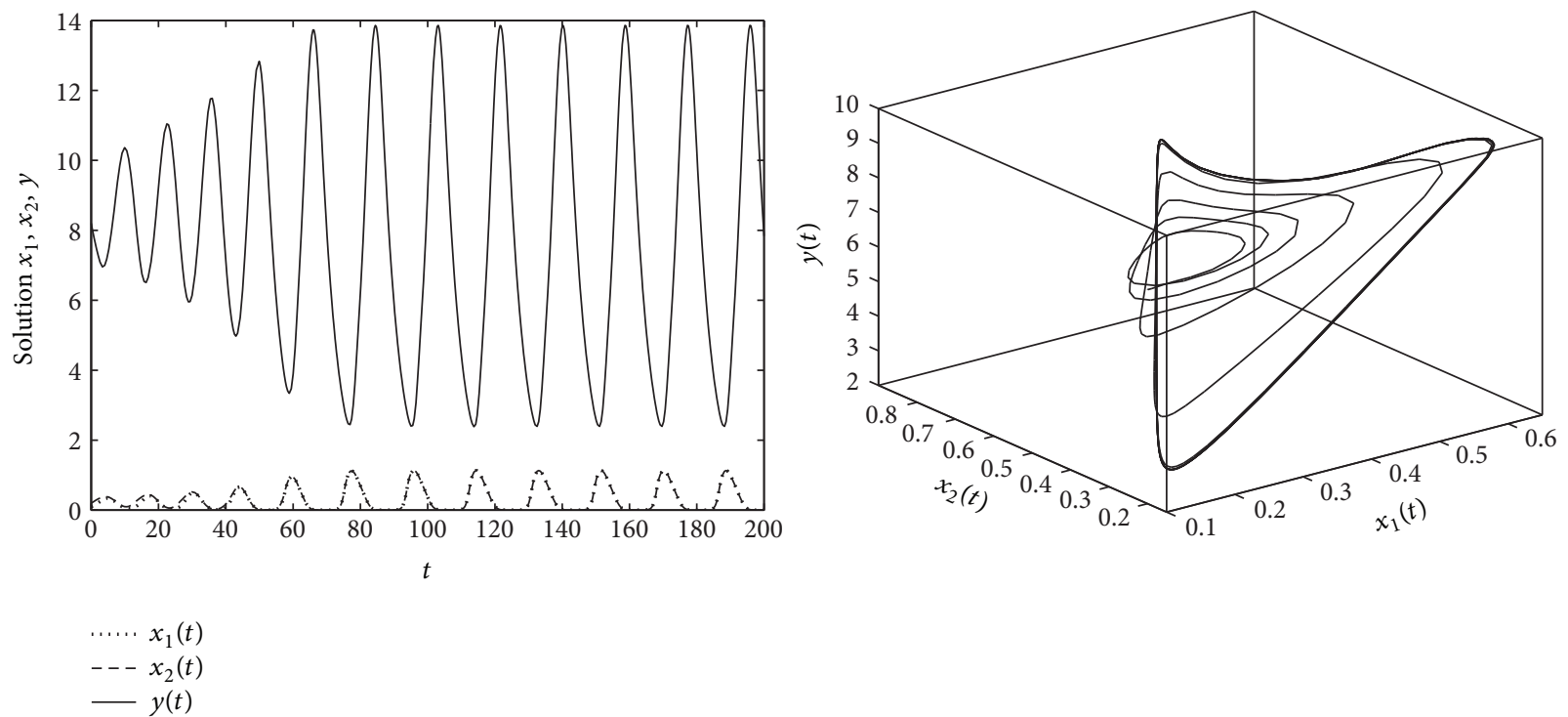

FIGURE 8: The behavior of $x_{1}, x_{2}$, and $y$ with respect to $t$ for equilibrium $E_{2}^{2}$ with $\tau=2.6$.

example, what occurs at the first two equilibria $E_{2}^{1}$ and $E_{2}^{2}$ is similar to the previous example where Hopf bifurcations occur at $\tau_{0}$ 's and the stable limit cycle exists. The predator and prey species coexist. As for the other equilibrium $E_{2}^{3}$, the system is locally asymptotically stable where predator and prey species also coexist. Finally, the bifurcation diagram for Example 7 is shown in Figure 10.

\section{Concluding Remarks}

In this paper, we find that system (7) has complex dynamics behavior. By Theorem 2, our results show that the predator and prey coexist permanently if $d<g \alpha x_{1}^{*}\left(x_{1}^{*}-1\right) /\left(1+g\left(x_{1}^{*}-\right.\right.$ $1)$ ); that is, the adult predators' reproductive rate at the peak of prey abundance is larger than its death rate. On the other hand, the predator faces extinction, if $d>g \alpha x_{1}^{*}\left(x_{1}^{*}-1\right) /(1+$ $\left.g\left(x_{1}^{*}-1\right)\right)$, which implies that the predator's possible highest reproductive rate is less than its death rate. We also find the stability switches of the positive equilibrium $E_{2}$ due to the increase of $\tau$. Our results show that when there is no time delay or the time delay is very small, the positive equilibrium $E_{2}$ is locally asymptotically stable. As the time delay increases to the critical value, it can cause a stable equilibrium to become unstable and Hope bifurcation can occur. 

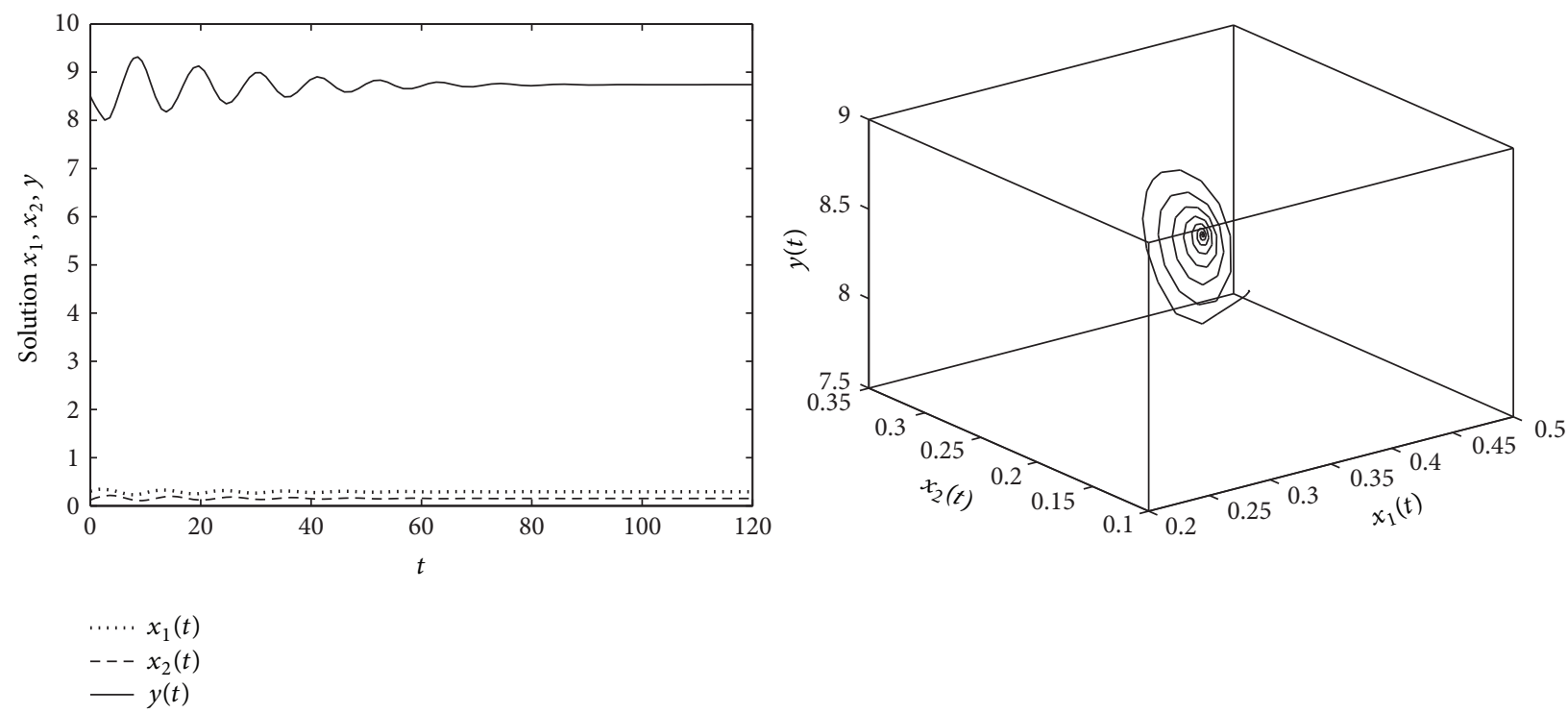

FIGURE 9: The behavior of $x_{1}, x_{2}$, and $y$ with respect to $t$ for equilibrium $E_{2}^{3}$ with $\tau=2.6$.

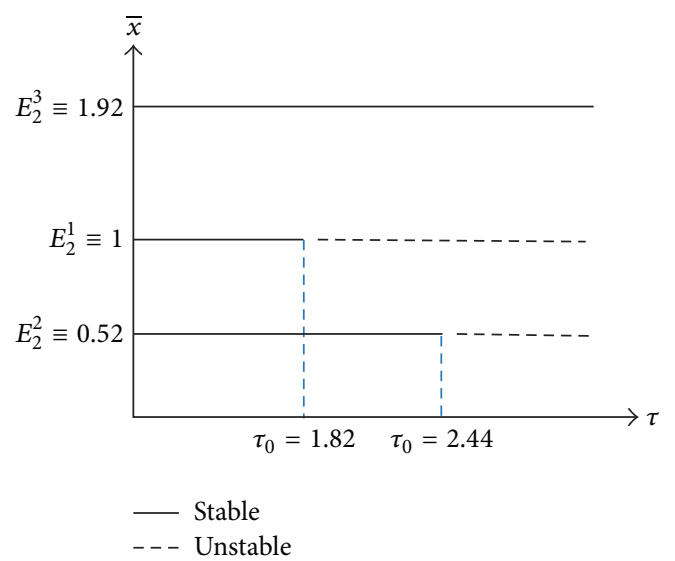

Figure 10: Bifurcation diagram for Example 7.

\section{Conflicts of Interest}

The author declares that there are no conflicts of interest regarding the publication of this article.

\section{Acknowledgments}

This research was supported by Chiang Mai University, Thailand.

\section{References}

[1] C. Celik, "The stability and Hopf bifurcation for a predator-prey system with time delay," Chaos, Solitons and Fractals, vol. 37, no. 1, pp. 87-99, 2008.
[2] H. I. Freedman and S. G. Ruan, "Hopf bifurcation in threespecies food chain models with group defense," Mathematical Biosciences, vol. 111, no. 1, pp. 73-87, 1992.

[3] S. B. Hsu and T. W. Huang, "Global stability for a class of predator-prey systems," SIAM Journal on Applied Mathematics, vol. 55, no. 3, pp. 763-783, 1995.

[4] S.-B. Hsu and T.-W. Hwang, "Hopf bifurcation analysis for a predator-prey system of Holling and Leslie type," Taiwanese Journal of Mathematics, vol. 3, no. 1, pp. 35-53, 1999.

[5] Z. Lu and X. Liu, "Analysis of a predator-prey model with modified Holling-Tanner functional response and time delay," Nonlinear Analysis. Real World Applications. An International Multidisciplinary Journal, vol. 9, no. 2, pp. 641-650, 2008.

[6] Y. Song and S. Yuan, "Bifurcation analysis in a predatorprey system with time delay," Nonlinear Analysis. Real World Applications. An International Multidisciplinary Journal, vol. 7, no. 2, pp. 265-284, 2006.

[7] M. Tansky, "Switching effect in prey-predator system," Journal of Theoretical Biology, vol. 70, no. 3, pp. 263-271, 1978.

[8] Q. J. Khan, E. Balakrishnan, and G. C. Wake, "Analysis of a predator-prey system with predator switching," Bulletin of Mathematical Biology, vol. 66, no. 1, pp. 109-123, 2004.

[9] W. W. Murdoch, "Switching in general predators: experiments on predator specificity and stability of prey populations," Ecological Monographs, vol. 39, no. 4, pp. 335-364, 1969.

[10] Prajneshu and P. Holgate, "A prey-predator model with switching effect," Journal of Theoretical Biology, vol. 125, no. 1, pp. 6166, 1987.

[11] E. Teramoto, K. Kawasaki, and N. Shigesada, "Switching effect of predation on competitive prey species," Journal of Theoretical Biology, vol. 79, no. 3, pp. 303-315, 1979.

[12] Y. Saito and Y. Takeuchi, "A time-delay model for prey-predator growth with stage structure," Canadian Applied Mathematics Quarterly, vol. 11, no. 3, pp. 293-302, 2003.

[13] W. G. Aiello and H. I. Freedman, "A time-delay model of singlespecies growth with stage structure," Mathematical Biosciences, vol. 101, no. 2, pp. 139-153, 1990. 
[14] W. G. Aiello, H. I. Freedman, and J. Wu, "Analysis of a model representing stage-structured population growth with statedependent time delay," SIAM Journal on Applied Mathematics, vol. 52, no. 3, pp. 855-869, 1992.

[15] Y. Qu and J. Wei, "Bifurcation analysis in a time-delay model for prey-predator growth with stage-structure," Nonlinear Dynamics. An International Journal of Nonlinear Dynamics and Chaos in Engineering Systems, vol. 49, no. 1-2, pp. 285-294, 2007.

[16] M. Y. Li and H. Shu, "Global dynamics of a mathematical model for HTLV-I infection of CD4 ${ }^{+} \mathrm{T}$ cells with delayed CTL response," Nonlinear Analysis: Real World Applications, vol. 13, no. 3, pp. 1080-1092, 2012. 


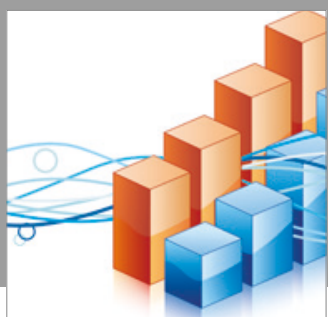

Advances in

Operations Research

vatersals

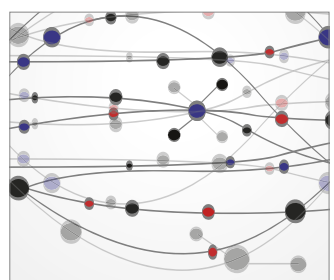

\section{The Scientific} World Journal
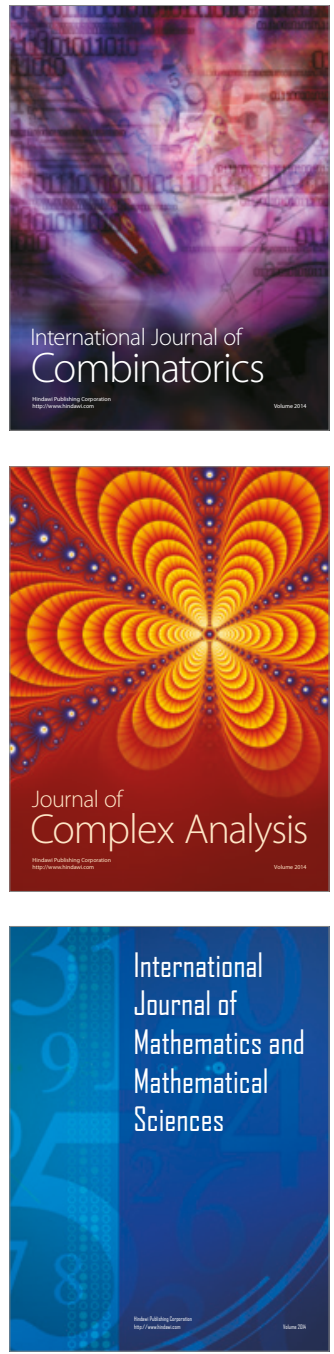
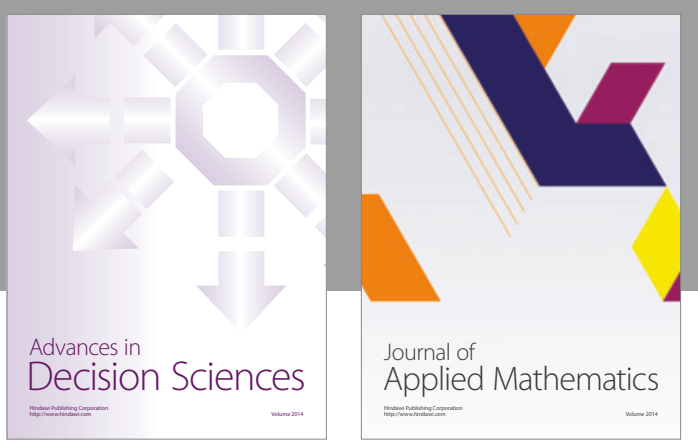

Algebra

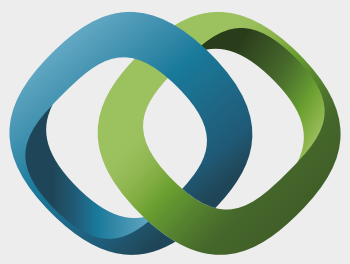

\section{Hindawi}

Submit your manuscripts at

https://www.hindawi.com
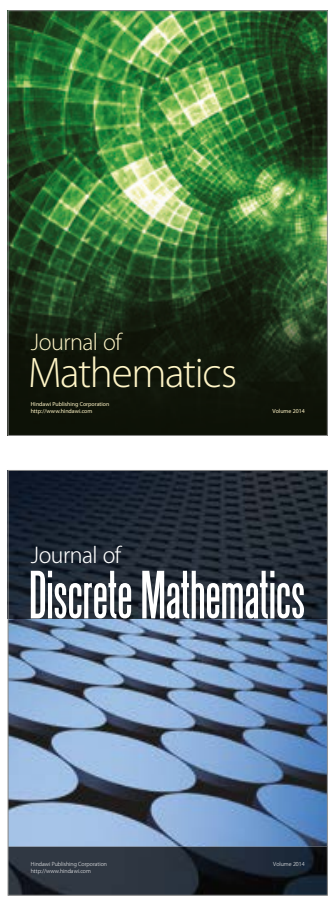

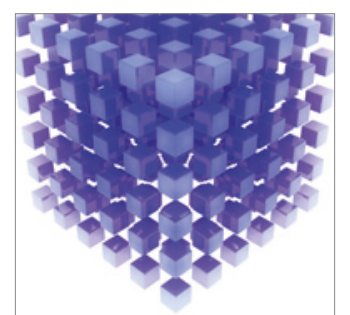

Mathematical Problems in Engineering
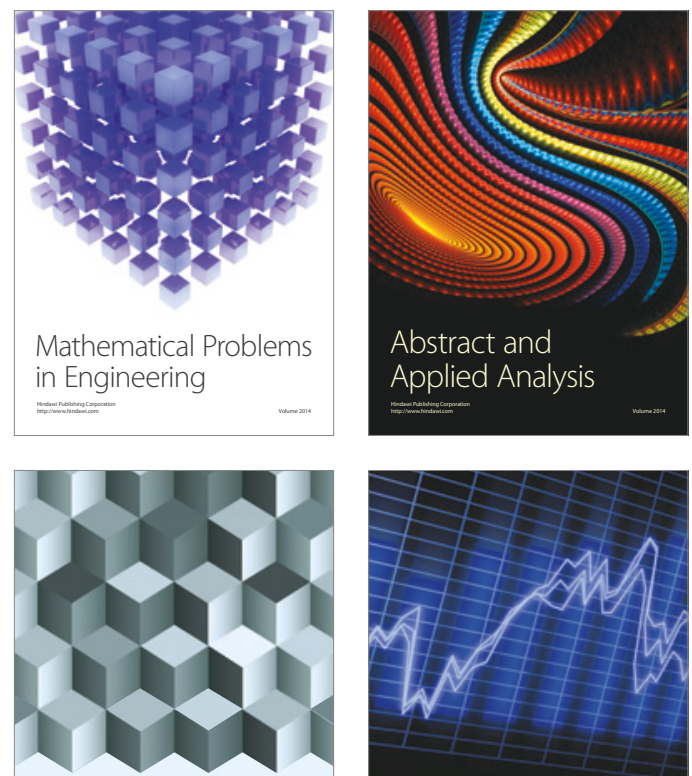

Journal of

Function Spaces

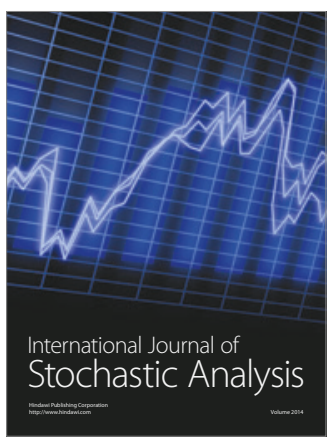

Probability and Statistics
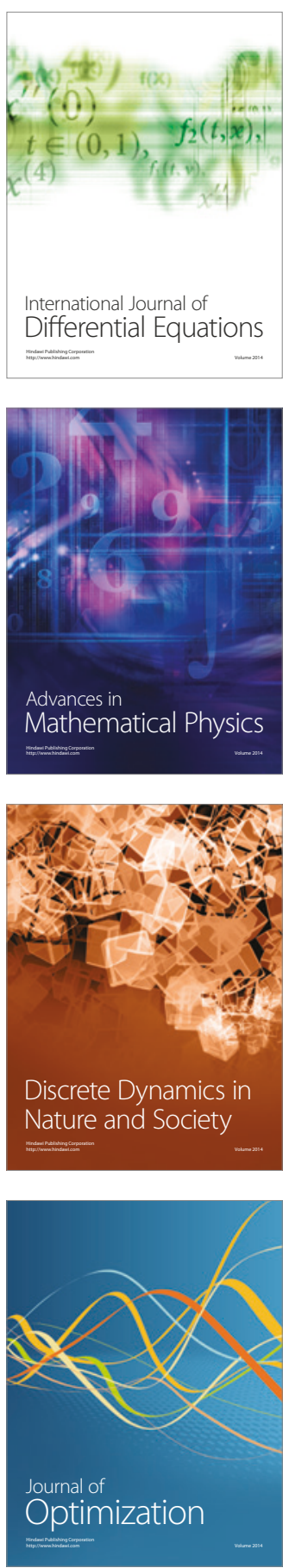